\title{
Es ist höchste Zeit für die Volksinitiative «JA zur Hausarztmedizin»!
}

Peter Tschudi

Präsident des Initiativkomitees

\begin{abstract}
Unterschreiben Sie heute!
Diesem Heft ist eine Unterschriftenkarte zur Volksinitiative beigelegt.

Bitte beachten Sie beim Sammeln von Unterschriften Folgendes: Unterschriftsberechtigt sind nur Schweizer Bürgerinnen und Bürger über 18 Jahren. Auf einem Unterschriftenbogen/-karte dürfen nur Personen der gleichen politischen Wohngemeinde unterschreiben.

Wünschen Sie weitere Unterlagen oder Auskünfte, wenden Sie sich bitte an: Eidgenössische Volksinitiative «Ja zur Hausarztmedizin», Postfach 8319, 3001 Bern, Tel. 03156000 26, www.jzh.ch
\end{abstract}

Die Schweiz bewegt sich derzeit ungebremst in Richtung eines massiven Hausärztemangels. Die Schere zwiebot und Nachfrage in der Hausarztmedizin Hausarztmedizin langfristig sichern und fördern.

Seit der Publikation des Initiativtextes im Bundesblatt am 29.9.2009 und der offiziellen Lancierung am 1.10.2009 läuft nicht nur die offizielle 18-monatige Sammelfrist. Es ist gleichzeitig das erste Grossprojekt von «Hausärzte Schweiz» und der bisher grösste Politvorstoss in der Geschichte der Schweizer Hausärzte

Fazit der Vorbereitungsphase: Die hausärztlichen Reihen sind geschlossen! Alle bisherigen Grundversorgergesellschaften (SGAM, SGIM, SGP), die sich am

17. September 2009 zum neuen Dachverband «Hausärzte Schweiz» vereinigt haben, unterstützen die Initiative. Auch die FMH steht hinter dem Projekt.

Das Initiativkomitee besteht aus 26 Hausärztinnen und Hausärzten. Dies ist Teil der Strategie, die den Hausärztinnen und Hausärzten die Hauptrolle zuweist und deutlich macht, dass die Hausärzte das Heft in die eigene Hand genommen haben. Aber: Die Politiker, Interessensvertreter und Freunde der Hausarztmedizin sind natürlich herzlich eingeladen, im Unterstützungskomitee mitzuarbeiten!

Ein weiterer Teil der Strategie ist die sehr kurze Sammelzeit von viereinhalb Monaten: Unser erklärtes Ziel ist, am 1. April 2010 - am Tag der Hausarztmedizin - die notwendigen beglaubigten 100000 Unterschriften einzureichen. Damit wollen wir auf die Dringlichkeit des Anliegens aufmerksam machen. Denn es geht um nichts Geringeres als das Überleben der Hausarztmedizin!

Prof. Dr. med. Peter Tschudi Vorsteher Institut für Hausarztmedizin IHAMB

Universität Basel Praxis Hammer Bläsiring 160

CH-4057 Basel Tel. 0616928811

peter.tschudi@unibas.ch

www.ihamb.unibas.ch

\section{Die Schweiz hat zu wenig Hausärztinnen und Hausärzte!}

Spätestens seit der grossen Hausärztedemonstration in Bern vom 1. April 2006 wissen es alle: Die Schweiz hat zuwenig Hausärztinnen und Hausärzte. Wir bewegen uns ungebremst in Richtung eines massiven Haus- ärztemangels, und niemand in der Gesundheitspolitik war bisher ernsthaft willens noch in der Lage, darauf zu reagieren. Es fehlt schon aktuell an Nachwuchs in der Hausarztmedizin. Aus der repräsentativen Studie «Workforce 2005» wissen wir, dass bis 2016 die Hälfte der heute praktizierenden Hausärztinnen und Hausärzte in Pension gehen werden. Bis 2021 werden es gar 75 Prozent sein (Quelle: Workforce 2005 IHAMB). Das bedeutet: Bis in sieben Jahren werden rund 3200 und bis in 12 Jahren rund 4700 neue Hausarztpersonen benötigt, die das gleiche Pensum leisten wie die abtretenden Hausärztinnen und Hausärzte, nur um den Status quo der jetzigen Grundversorgung aufrechterhalten zu können - ohne Berücksichtigung der Bevölkerungsentwicklung! Aus den Berufszielstudien des Institutes für Hausarztmedizin Basel IHAMB (20022008) wissen wir hingegen, dass nur etwa $10 \%$ aller Medizinstudierenden das Berufsziel «Hausarzt/ärztin» angeben, und dass die Mehrheit davon (60-70\%) Frauen sein werden. Der klassische «Hausarzt» von morgen wird somit weiblich sein, eine deutlich kürzere Lebensarbeitszeit haben und Teilzeit arbeiten.

\section{Was ist zu tun?}

Wir müssen nicht nur die Aus- und Weiterbildung zum Hausarzt bzw. zur Hausärztin attraktiv gestalten, wir müssen ganz generell den Beruf «Hausärztin/Hausarzt» attraktiver machen, indem wir ihn aufwerten. Wir müssen die diagnostischen, therapeutischen und präventiven Möglichkeiten in der Praxis, im Labor, im Röntgen, beim Ultraschall und beim EKG usw. vielseitiger und interessanter gestalten. Und das geht nicht ohne Investitionen, und zwar von allen.

Ohne funktionierende Hausarztmedizin können wir die Grundversorgung der Bevölkerung in naher Zukunft nicht mehr sicherstellen. Nur mit einer effizienten Hausarztmedizin wird unser Gesundheitswesen besser und kostengünstiger - zum Wohl der Bevölkerung!

\section{Fazit}

Dies ist der Hintergrund für die Volksinitiative «JA zur Hausarztmedizin». Sie ist der Schlüssel für eine nachhaltige Hausarztmedizin und sie zeigt den Weg, wie die medizinische Grundversorgung unserer Bevölkerung langfristig gesichert werden kann. Deshalb liegt diese Initiative im Interesse der ganzen Bevölkerung, primär der Patientinnen und Patienten.

Helfen Sie bitte mit und unterschreiben auch Sie diese Initiative - auf der beiliegenden Unterschriftenkarte! Vielen Dank! 